www.nature.com/ejhg

\title{
A genome scan for loci influencing anti-atherogenic serum bilirubin levels
}

\author{
Florian Kronenberg ${ }^{\star 1,2}$, Hilary Coon ${ }^{3}$, Alexander Gutin ${ }^{4}$, Victor Abkevich ${ }^{4}$, \\ Mark E Samuels ${ }^{4,5}$, Dennis G Ballinger ${ }^{4,6}$, Paul N Hopkins ${ }^{1}$ and Steven C Hunt ${ }^{1}$
}

${ }^{1}$ Cardiovascular Genetics, University of Utah, Salt Lake City, Utah, USA; ${ }^{2}$ Institute of Medical Biology and Human Genetics, University of Innsbruck, Innsbruck, Austria; ${ }^{3}$ Department of Psychiatry, University of Utah, Salt Lake City, Utah, USA; ${ }^{4}$ Myriad Genetics, Inc., Salt Lake City, Utah, USA

Epidemiological studies have shown an association of decreased serum bilirubin levels with coronary artery disease. Two segregation analyses in large pedigrees have suggested a major gene responsible for high bilirubin levels occurring in about $12 \%$ of the population. Based on a recessive model from a previous segregation analysis, we performed a genome scan using 587 markers genotyped in 862 individuals from 48 Utah pedigrees to detect loci linked to high bilirubin levels. As a complementary approach, non-parametric linkage (NPL) analysis was performed. These two methods identified four regions showing evidence for linkage. The first region is on chromosome 2q34-37 with multipoint LOD and NPL scores of 3.01 and 3.22, respectively, for marker D2S1363. This region contains a previously described gene, uridine diphosphate glycosyltransferase 1, which has been associated with high bilirubin levels. A polymorphism in the promoter of this gene was recently shown to be responsible for Gilbert syndrome which is associated with mild hyperbilirubinemia. The other regions were found on chromosomes 9q21, 10q25-26, and 18q12 with maximum NPL scores of 2.39, 1.55, and 2.79, respectively. Furthermore, we investigated in these pedigrees the association between bilirubin levels and coronary artery disease. One-hundred and sixty-one male and $\mathbf{4 1}$ female subjects had already suffered a coronary artery disease event. Male patients showed significantly lower bilirubin concentrations than age-matched controls. This association, however, was not observed in females. These results provide evidence that loci influencing bilirubin variation exist on chromosomes $2 q 34-37,9 q 21,10 q 25-26$, and $18 q 12$ and confirms the association of low bilirubin levels with coronary artery disease in males. European Journal of Human Genetics (2002) 10, 539-546. doi:10.1038/sj.ejhg.5200842

Keywords: bilirubin; linkage analysis; genetics; antioxidants; atherosclerosis; coronary artery disease

\section{Introduction}

There is increasing support that the bile pigment bilirubin formed during haeme catabolism has antioxidative and cytoprotective properties. $^{1}$ Most of this evidence comes

\footnotetext{
*Correspondence: Florian Kronenberg; Institute of Medical Biology and Human Genetics, University of Innsbruck, Schöpfstr. 41, A-6020 Innsbruck, Austria. Tel: (+43) 512 507-3474; Fax: (+43) 512 507-2861; E-mail: Florian.Kronenberg@uibk.ac.at

Current addresses: ${ }^{5}$ Xenon Genetics, Inc., Vancouver, BC, Canada;

${ }^{6}$ Ingenuity Systems, Inc., Alviso, California, USA

Received 21 September 2001; revised 14 May 2002; accepted 16 May 2002
}

from in vitro investigations ${ }^{2-11}$ and is in line with epidemiological studies describing lower bilirubin concentrations in patients with coronary artery disease $(\mathrm{CAD})^{12-18}$ or in offspring with a parental history of heart attack. ${ }^{19}$ A major gene controlling high bilirubin levels has been implicated by two recent segregation analyses. A study in 1240 adults in 84 Utah pedigrees indicated that a major gene is responsible for elevated bilirubin levels in about $12 \%(q=0.34)$ of the subjects. This major gene explained about $27 \%$ of the variance in bilirubin levels. ${ }^{13}$ Similar results were found in the NHLBI Family Heart Study in 555 families of 1292 individuals. $^{14}$ The most parsimonious model in both studies 
was a recessive model for high bilirubin levels and a significant polygenic effect.

The present study describes the first genome-wide linkage analysis for loci for high bilirubin levels in 48 large pedigrees with the same ethnicity as investigated in a previous segregation analysis. ${ }^{13}$

\section{Subjects}

This study is based on 1735 individuals (912 men and 823 women) in 48 pedigrees ascertained because of two or more first-degree relatives with early cardiovascular disease. The minimum and maximum pedigree sizes were 15 and 117 persons, respectively. Serum bilirubin levels and genotypes for linkage analysis were available in 877 of the 1735 Caucasian subjects. Their mean age and body mass index was $39 \pm 19$ years and $27.0 \pm 7.0 \mathrm{~kg} / \mathrm{m}^{2}$, respectively. The major proportion of subjects with unknown phenotype and genotypes were individuals in the upper generations of the multigenerational families who were already deceased but were needed to connect the pedigrees together.

Families were ascertained by discharge histories from the hospital $^{20}$ and by the Utah Health Family Tree Project. ${ }^{21}$ Early cardiovascular disease was defined as the onset of at least one of the following conditions before the age of 56 years for males or before the age of 66 years for females: myocardial infarction (MI), coronary bypass surgery (CABG), coronary angioplasty (PTCA) and unstable angina pectoris.

Families who met the study criteria were sent a personal health questionnaire which contained questions on demographics, family history, anthropometrics, smoking habits, alcohol consumption, physical exercise, medications as well as hospitalization for cardiovascular-related diseases. Participants visited the University of Utah Cardiovascular Genetics Research Clinic for a blood draw after overnight fast, anthropometric measurements (height, weight, elbow diameter, abdominal and hip measurements and two sitting blood pressure measurements), a physical examination and an interview on cardiovascular history, stroke history, cardiovascular and respiratory symptoms, other medical conditions, illnesses and present therapy. Subjects were excluded from the analysis when they were not fasting, had abnormal liver or kidney function or when they had a history of kidney disease.

\section{Bilirubin measurement}

Total serum bilirubin was measured by a thin film adaptation of a diazonium salt, colorimetric method using the Vitros analyser (Ortho-Clinical Diagnostics, Inc. Rochester NY 14650, USA). In this method, dyphylline is used to dissociate unconjugated bilirubin from albumin. Then, unconjugated bilirubin, conjugated bilirubin, and albumin-linked bilirubin (delta) react with the diazonium salt 4-(N-carboxymethylsulfamyl) benzenediazonium hexa- fluorophosphate to produce azobilirubin chromophores which are read spectrophotometrically at 540 and 460 nм. $^{22}$

\section{Genotypes}

DNA was extracted from frozen buffy coats using standard methods. For the genome search, 583 autosomal and four gonosomal markers with an average spacing of $5.7 \mathrm{cM}$ were genotyped at Myriad Genetics, Inc. Most of the markers are trinucleotide or tetranucleotide repeats and are described in public databases such as Marshfield (http://research. marshfieldclinic.org/genetics), Genethon (http://www. genethon.fr) or Human Genome Database (GDB; http:// gdbwww.gdb.org). PCR products were analysed on ABI 377 fluorescent sequencing machines using standard techniques. In-house allele calling programmes and extensive quality control algorithms were used to ensure high quality genotypes. Inheritance of all alleles was verified using the program Pedcheck. ${ }^{23}$ The genetic map used for all analysis and tables was internally generated from a larger superset of Utah pedigrees by Myriad Genetics, Inc., using the CRIMAP program ${ }^{24}$ on 3916 meioses and closely corresponds to the Marshfield map.

\section{Segregation and linkage analysis}

Parametric linkage analysis was performed using bilirubin levels as a quantitative trait after adjusting for age, age ${ }^{2}$, age $^{3}$, and sex. Fifteen of the 877 subjects who had adjusted bilirubin levels above the mean +5 standard deviations $(=38.04 \mu \mathrm{mol} / \mathrm{l})$ were excluded from the analysis. Recessive model parameters including the estimated gene frequency of $q=0.34$ and genotype means of 9.4 and $16.9 \mu \mathrm{mol} / 1$ were obtained from a previous segregation analysis. ${ }^{13}$ We rely on the model parameters of this recent study rather than on those from the present one because the pedigrees in the present study were not expanded by usual sequential sampling rules and persons with CAD or lipid abnormalities were preferentially genotyped. The previous study, however, ascertained pedigree members independently of whether some branches were associated with CAD or dyslipidemia. The disease-driven approach might be advantageous for linkage analysis but is prone to disturb segregation analysis. Nevertheless, we also performed maximum likelihood segregation analysis on the present pedigrees using the Pedigree Analysis Package (PAP) ${ }^{25}$ and compared the results for the four most important regions with parameter estimates from both datasets.

Linkage analysis was performed using robust multipoint linkage statistics proposed by Göring and Terwilliger ${ }^{26}$ as implemented in Myriad Genetics' MCLINK software program. ${ }^{27}$ This programme was developed to perform multipoint analysis on very large pedigrees with any number of markers and is robust to deviations from a normal distribution and to model mis-specifications. It generates multipoint marker haplotypes using a blocked 
Gibbs sampling approach, checking that the solution converges to the same estimates after multiple runs. Multipoint plots of the best linkage region are produced showing maximum LOD scores calculated at each marker across the chromosome. The haplotype solutions generated by MCLINK $^{27}$ were also used for nonparametric linkage (NPL) statistics. $^{28}$ The method is similar to the method developed by Kruglyak et al. ${ }^{29}$ but is extended to the analysis of quantitative traits. The main differences between these two methods were discussed recently. ${ }^{30}$ NPL statistics can detect genes with causal mutations that segregate among individuals with either low or high bilirubin values. It not only circumvents the problem of model mis-specifications but also has the ability to recognize the effects of multiple mutations, even if their penetrances, phenotypic effects, and modes of inheritance are quite different. An asymptotic formula was derived to adjust NPL scores, such that equal LOD and NPL scores translated to the same $P$ values. ${ }^{30}$

\section{Other statistical methods}

We compared age and HDL cholesterol concentrations between CAD patients and controls by unpaired $t$-tests. Wilcoxon rank sum tests were used for bilirubin concentrations. Furthermore, we compared the bilirubin levels adjusted for age, age $^{2}$, age ${ }^{3}$ and smoking status by multiple regression analysis. Since the data included in this analysis were based on families and a correlation of the variables within the families was expected, a mixed linear model was used for adjustment to accommodate this intrafamilial correlation by using an identification number for each family as a random effect in the model. Variables predicting the CAD status were identified by stepwise logistic regression analysis.

\section{Results}

\section{Linkage analysis}

The means \pm SD bilirubin concentrations were $12.1 \pm 5.6$ $\mu \mathrm{mol} / 1$ in men and $9.7 \pm 3.9 \mu \mathrm{mol} / \mathrm{l}$ in women, respectively. Before bilirubin concentrations were used for linkage analysis they were adjusted for age, age ${ }^{2}$, age $^{3}$ and sex, since these variables showed significant correlations with bilirubin concentrations (all $P$ values $<0.0001$ using GLM procedure). The only other covariate in Table 1 which significantly correlated with bilirubin levels was albumin. However, we did not adjust for albumin since this correlation can be explained by the binding protein function of albumin for bilirubin. Table 1 shows the clinical characteristics of the 862 study subjects who had bilirubin levels and genotypes determined and were used for linkage analysis.

The results from parametric linkage analysis are based on a recently described recessive model $^{13}$ considering 587 markers. We repeated the analysis for chromosomes that showed LOD scores above 2.0 after estimating the model parameters from the pedigrees used in this linkage analysis. These pedigrees favoured a codominant rather than a reces- sive model. The genetic model parameters of the recessive and codominant models are provided in Table 2 . MCLINK ${ }^{27}$ was applied to determine the marker-segregation pattern and the generated haplotype solutions were used to generate model-based multipoint LOD scores and non-parametric NPL scores. ${ }^{28}$ Forty-four markers showed either a multipoint LOD score or an NPL score greater than 1 (Table 3). Only four chromosomes harbored three or more markers with LOD and/or NPL scores above 1 . The results for these chromosomes are presented in Figure 1 and Table 3 .

The highest multipoint LOD scores and NPL scores were found for markers on the long arm of chromosome 2 (2q34-37). Nine markers in a region of about $60 \mathrm{cM}$ showed LOD scores above one. NPL statistics identified six markers in this region with NPL scores above one. The highest NPL scores were obtained for markers D2S434 (3.29) and D2S1363 (3.22) within an intermarker distance of about $11 \mathrm{cM}$. The results from parametric analysis revealed a LOD score of 3.01 for marker D2S1363 using the recessive model parameters (Figure 1 and Table 3). When the parametric analysis was repeated using the codominant parameters, a LOD score of 2.51 was obtained.

The second most interesting region was observed on chromosome 18. Depending on the analysis method used, two different regions were identified which are suggestive for linkage. NPL scores identified a region around $50 \mathrm{cM}$ with NPL scores of 2.79 (D18S974) and 2.10 (D18S535) (18q12). Parametric linkage analysis, however, did not provide positive LOD scores for this region. Instead, this method revealed LOD scores around 2.2 for the telomeric region on the short arm of chromosome 18 (18p11) (Figure 1 and Table 3). When this analysis was repeated using the codominant model parameters LOD scores increased to 2.60 (D18S976)

A third region linked to high bilirubin levels was located on the long arm of chromosome 10 (10q25-26) with seven markers with LOD scores mostly between 2 and 2.5 in the terminal $45 \mathrm{cM}$. These scores decreased when either parameters from the codominant model (D10S1237: LOD $=1.11$ ) or NPL scores were analysed (D10S1237: NPL score=1.55).

NPL analysis revealed a $7 \mathrm{cM}$ region on chromosome 9 (9q21) which included four markers with NPL scores between 2.1 and 2.4. LOD scores remained below 1.0 when parametric analysis was performed with either recessive or codominant parameters.

Other markers which showed LOD or NPL scores above 1.0 were single observations which were usually not supported by positive scores of adjacent markers.

\section{Association of bilirubin with CAD}

Since this study is based on pedigrees ascertained for two or more first-degree relatives with early CAD, it allowed us to compare bilirubin levels between subjects with and without CAD. We matched 161 male patients with CAD and twice 
Table 1 Clinical characteristics of the 862 study subjects who had bilirubin levels and genotypes determined and who were used for linkage analysis

\begin{tabular}{lc}
\hline Variable & Mean \pm SD or \% \\
\hline Age & $39 \pm 19$ \\
Male sex & $50.3 \%$ \\
BMl, $\mathrm{kg} / \mathrm{m}^{2}$ & $27.0 \pm 7.0$ \\
Bilirubin, $\mu \mathrm{mol} / \mathrm{l}$ & $10.9 \pm 4.9$ \\
Total protein, g/dl & $7.1 \pm 0.4$ \\
Albumin, g/dl & $4.2 \pm 0.4$ \\
Plasma glucose, mg/dl & $89 \pm 24$ \\
Uric acid, mg/dl & $5.5 \pm 1.5$ \\
Total cholesterol, mg/dl & $192 \pm 50$ \\
HDL cholesterol, mg/dl & $39 \pm 14$ \\
LDL cholesterol, mg/dl & $112 \pm 38$ \\
Triglycerides, mg/dl & $199 \pm 138$ \\
Serum creatinine, mg/dl & $0.87 \pm 0.24$ \\
Systolic blood pressure, $\mathrm{mm} \mathrm{Hg}$ & $122 \pm 19$ \\
Diastolic blood pressure, $\mathrm{mm} \mathrm{Hg}$ & $75 \pm 11$ \\
Diabetes mellitus, \% & $4.15 \%$ \\
Current smokers, \% & $9.0 \%$ \\
\hline
\end{tabular}

Table 2 Genetic model parameters of bilirubin levels used for linkage analysis

\begin{tabular}{lcc}
\hline & Recessive model & Codominant model \\
\hline $\mathrm{q}$ & 0.34 & 0.22 \\
$\mu_{1}$ & 9.4 & 9.3 \\
$\mu_{2}$ & $9.4\left(=\mu_{1}\right)$ & 10.7 \\
$\mu_{3}$ & 16.9 & 23.9 \\
$\sigma$ & 2.9 & 3.4 \\
\hline
\end{tabular}

q... gene frequency, $\mu_{1}-\mu_{3} \ldots$ means for each of the three genotypes, $\sigma \ldots$ standard deviation. Recessive model parameters were obtained from a previous segregation analysis. ${ }^{13}$ Linkage analysis was repeated on the four most important regions using parameter estimates from maximum likelihood segregation analysis on the present pedigrees which favoured a codominant model.

the number of male controls for age and observed significantly lower concentrations in patients $(11.3 \pm 4.6 \mathrm{vs}$ $12.7 \pm 5.3 \mu \mathrm{mol} / 1, \quad P=0.005$ ) (Table 4). Logistic regression analysis showed HDL cholesterol $(P=0.009)$ and bilirubin concentrations $(P=0.043)$ to be significantly associated with CAD. Furthermore, we matched five times the number of female controls for age to the 41 female patients with CAD and observed a tendency to lower bilirubin levels in CAD patients which, however, did not reach statistical significance (Table 4).

\section{Discussion}

\section{Evidence for anti-atherogeneity of bilirubin}

Previous epidemiological studies described an association between low bilirubin levels and coronary heart disease. ${ }^{12-19}$ It was found that plasma bilirubin levels correlated inversely with known CAD risk factors, such as smoking, LDL cholesterol, diabetes, and obesity, and correlated directly with HDL cholesterol. ${ }^{19,31}$ The relation between low bilirubin levels and CAD, however, remained significant after adjustment for known CAD risk factors. ${ }^{16,31}$ This association was further confirmed in the prospective British Regional Heart Study that observed 737 major ischemic heart disease events in 7685 middle-aged men during 11.5 years of follow-up. ${ }^{15}$ A small case-control study found decreased values of bilirubin indicative of the presence of atherosclerosis in smokers. ${ }^{32}$ The Family Heart Study pointed to a gender-specific effect of bilirubin on CAD since it observed an association of borderline significance in males but not in females. ${ }^{14}$ This is in accordance with our results showing only significant differences for bilirubin levels in males but not in females (Table 4) and with results from the prospective Framingham Offspring Study. The latter suggested higher bilirubin levels to be associated with a lower cardiovascular risk in men, but the pattern was unclear in women. ${ }^{18}$ Ten-year follow-up mortality data from Belgium observed an inverse association between bilirubin levels and all-cause and cancer mortality but not cardiovascular mortality in men. None of these associations was observed in women. ${ }^{33}$

The association of bilirubin with atherosclerosis might be explained by in vitro findings showing antioxidative and

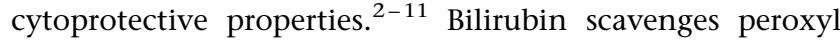
radicals and suppresses the oxidation in liposomes more efficiently than alpha-tocopherol. ${ }^{2}$ The antioxidative properties may be independent of whether bilirubin is unconjugated, conjugated, free, or albumin-bound. ${ }^{3,4,6}$ Recent investigations proposed bilirubin to be an antiatherogenic endproduct in the heme-oxygenase-carbon monoxide signalling pathway. ${ }^{9,34-36}$ The cytoprotective properties of bilirubin may be explained by its inhibition of protein kinase $\mathrm{C}$ (PKC). ${ }^{10,11,37}$ Haeme oxygenase activity is related to a faster resolution of inflammation, whereas the inhibition of this enzyme seems to increase the inflammatory response. ${ }^{38}$

\section{Familial aggregation and linkage analysis for high} bilirubin levels

Familial aggregation of bilirubin levels was supported by recent segregation analysis on 1240 adults in 84 Utah pedigrees suggesting a major gene controlling bilirubin levels. ${ }^{13}$ The parameters obtained from the segregation model were used in the linkage analysis. It was estimated that the major gene was responsible for elevated bilirubin levels in $12 \%$ ( $q=0.34$ ) of the subjects explaining $27 \%$ of the variance in bilirubin levels. ${ }^{13}$ An ascertainment correction was not possible because the CAD proband founders of the pedigrees were deceased. Therefore our estimated gene frequency for high, protective bilirubin levels might be lower than in a random population since these CAD pedigrees might have lower rather than elevated bilirubin levels. A segregation model for bilirubin in the Family Heart Study whose families were ascertained half at random and half for familial CAD and in which ascertainment correc- 
Table 3 Markers with linkage scores above 1 in parametric (recessive model) and nonparametric linkage analysis

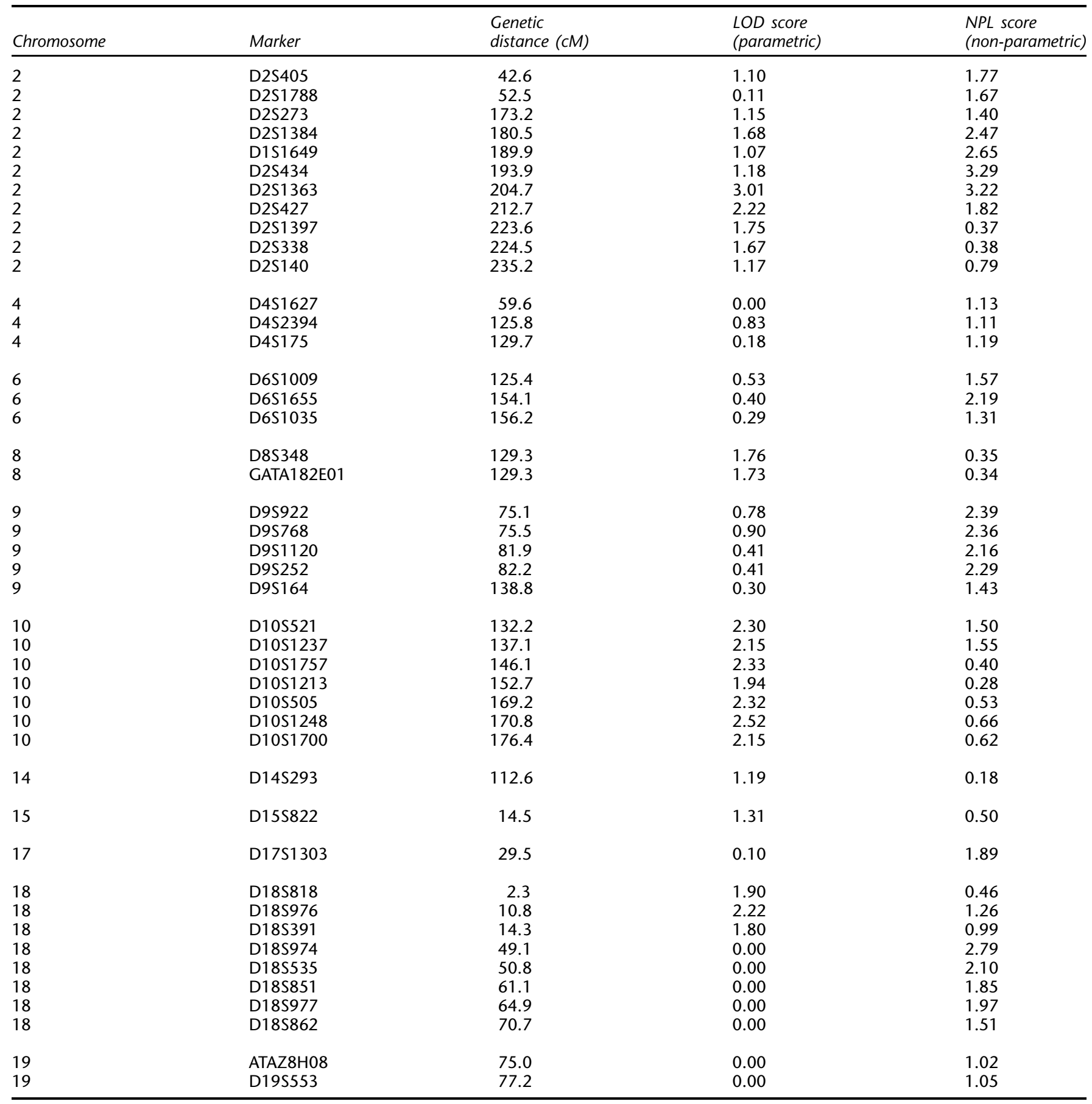

tion was possible showed a higher gene frequency $(q=0.48)$ but otherwise very similar parameters. ${ }^{14}$ The gene frequency estimate was even lower in the current set of pedigrees probably because there was an even greater selection of high-risk CAD families. Using the parameter estimates from the Utah pedigrees, ${ }^{13}$ we found three chro- mosomal regions that were linked to high bilirubin levels and a further region when we used model-free NPL statistics. The most important region is on chromosome 2 q3437 including marker D2S1363 (NPL score=3.22). This marker is approximately $12 \mathrm{cM}$ or $8 \mathrm{Mb}$ from an important candidate gene for high bilirubin levels which is uridine 

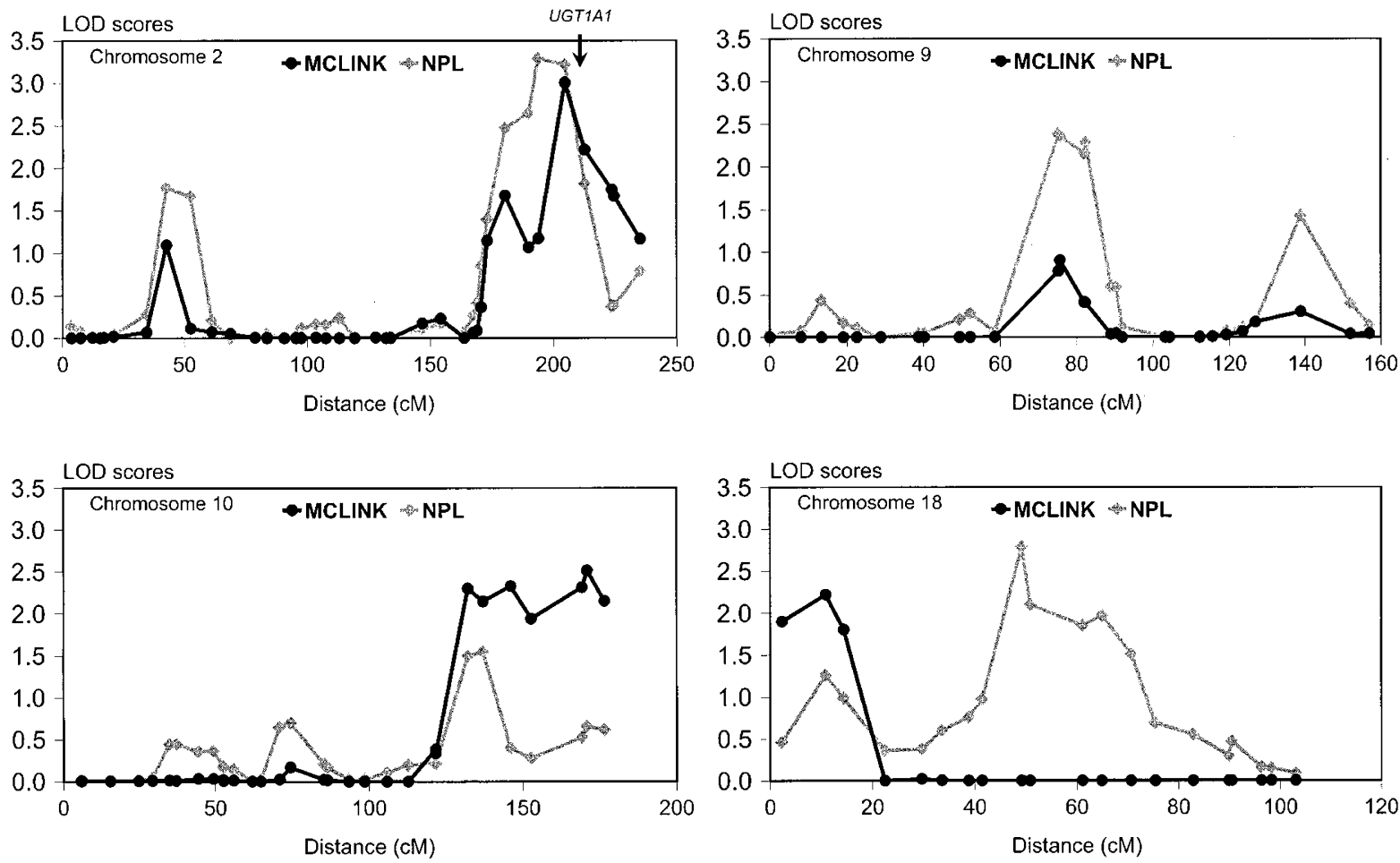

Figure 1 Suggestive regions for linkage with elevated bilirubin levels. Results represent multipoint LOD scores from analysis with MCLINK using a recessive model. Results from non-parametric linkage analysis (NPL) are shown as a complementary approach. The arrow in the Figure for chromsome 2 indicates the location of the most important candidate gene (UGT1A1: uridine diphosphate glycosyltransferase 1).

Table 4 Comparison of patients with coronary artery disease (CAD) and controls matched for age and stratified by gender

\begin{tabular}{lccl}
\hline & Controls & CAD patients & P value \\
\hline Men & & & \\
Number of subjects, $n$ & 322 & 161 & \\
Age, years & $58 \pm 11$ & $59 \pm 11$ & 0.47 \\
HDL cholesterol, mg/dl & $38.6 \pm 10.3$ & $36.3 \pm 9.7$ & 0.02 \\
Bilirubin, $\mu \mathrm{mol} / \mathrm{l}$ & $12.7 \pm 5.3$ & $11.3 \pm 4.6$ & 0.005 \\
Bilirubin adjusted, $\mu \mathrm{mol} /$ I $^{*}$ & $13.0 \pm 5.1$ & $11.8 \pm 4.4$ & 0.009 \\
& & & \\
Women & & & \\
Number of subjects, $n$ & 205 & 41 & \\
Age, years & $60 \pm 11$ & $60 \pm 11$ & 0.90 \\
HDL cholesterol, mg/dl & $54.7 \pm 16.5$ & $50.0 \pm 14.8$ & 0.09 \\
Bilirubin, $\mu$ mol/l & $9.9 \pm 3.5$ & $9.1 \pm 4.3$ & 0.16 \\
Bilirubin adjusted, $\mu \mathrm{mol} /$ I $^{*}$ & $10.0 \pm 3.3$ & $9.3 \pm 4.0$ & 0.18 \\
\hline
\end{tabular}

Values are presented as mean $\pm S D$; *adjusted for age, age $^{2}$, age ${ }^{3}$ and smoking status by multiple regression analysis using a mixed linear model.

diphosphate glycosyltransferase 1 (UGT1A1). ${ }^{39}$ Mutations in this gene are responsible for Crigler-Najjar syndrome $\mathrm{I}^{40-42}$ resulting in an absence of enzymatic bilirubin glucoronidation and Crigler-Najjar syndrome $\mathrm{II}^{43,44}$ with a severe deficiency of the enzyme. Both syndromes are very rare (1 in 1 million births) and are often but not always accompa- nied by a mild hyperbilirubinemia. A further syndrome with mild unconjugated hyperbilirubinemia is Gilbert syndrome which was suggested to have a frequency between 2 and 12\%. ${ }^{45-48}$ One of the most important polymorphisms responsible for this syndrome is a polymorphism in the promoter of the UGT1A1 gene. ${ }^{49}$ Promoters containing seven thymine adenine (TA) repeats have been reported to be less active than the wild-type six repeats, and the serum bilirubin levels of persons homozygous for seven repeats have been found to be higher than those with the wild-type six repeats. ${ }^{49-51}$ Bosma et al. reported a frequency of the seven repeat allele of about $40 \%{ }^{49}$ which is very close to the results from the previous segregation analysis in Utah pedigrees ${ }^{13}$ and in the NHLBI Family Heart Study. ${ }^{14}$ Whether this gene and/or other genes close to this gene are responsible for the linkage results for the high bilirubin levels remains to be determined in future studies. Interestingly, a recent study found a significantly lower prevalence rate of ischaemic heart disease in Gilbert syndrome when compared to a general population. ${ }^{52}$ This is in line with the inverse association between bilirubin and atherosclerosis found in this and several other studies. $^{12-19}$ Since NADH is involved in the metabolism of bilirubin, other candidates in this region are proteins and subcomplexes of the NADH dehydrogenase (NDUFS1, NDUFA10 and NDUFB3). Furthermore, CYP27A1 codes for 
the steroid 27-hydroxylase and is a cytochrome $\mathrm{P} 450$ enzyme of bile acid synthesis. ${ }^{53}$

The other regions suggestive for linkage are located on chromosomes 9q21, 10q25-26 and 18q12. No obvious candidate genes are available in these regions.

We repeated the analysis for chromosome 2, 9, 10 and 18 by using parameters obtained from segregation analysis of the pedigrees used for linkage analysis. Even if this analysis favoured a codominant rather than a recessive model, we observed for chromosomes 2, 9 and 18 very similar LOD scores. Parametric (LOD scores) and non-parametric (NPL scores) linkage analysis gave virtually the same results for chromosome 2 . The discordance between methods for other chromosomes might correspond to mis-specifications of the parametric model or reduced power of the nonparametric method.

\section{Methodological implications}

Despite initial enthusiasm about the concept of using linkage to identify genes related to common, multifactorial diseases or phenotypes, the results of many genome searches have been somewhat disappointing. The underlying heterogeneity of the measured phenotype and/or the small effects of any single gene on the phenotype may greatly reduce the power of linkage to identify such genes. This paper shows that there is a likelihood of success in identifying genes related to some phenotypes by linkage analysis. Two prior studies identified evidence for segregation of a major gene for bilirubin. This study shows that this major gene likely is located on chromosome 2. Polymorphisms in the promoter of the UGT1A1 gene, the most likely candidate within this region, are known to be associated with Gilbert syndrome causing hyperbilirubinemia and the associated gene frequency appears to be very close to the estimates from segregation analyses. These results and results from other linkage studies ${ }^{54,55}$ suggest that a linkage approach, especially in large pedigrees, may still be successful.

\section{Conclusions}

These results provide evidence that loci influencing bilirubin variation exist on chromosomes 2q34-37, 9q21, $10 \mathrm{q} 25-26$, and $18 \mathrm{q} 12$ and confirms the association of low bilirubin levels with coronary artery disease in males. Future studies have to narrow the region and should study the association of polymorphisms in the promoter of the UGT1A1 gene and CAD.

\section{Acknowledgements}

$F$ Kronenberg is supported by the 'Austrian Programme for Advanced Research and Technology' (APART) of the Austrian Academy of Science and by the Austrian Science Fund (P14717-GEN). This study was supported by a contract with Myriad Genetics, Inc. and a grant from NHLBI, HL21088.

\section{References}

1 Mayer M: Association of serum bilirubin concentration with risk of coronary artery disease. Clin Chem 2000; 46: 1723 -1727.

2 Stocker R, Yamamoto Y, McDonagh AF, Glazer AN, Ames BN: Bilirubin is an antioxidant of possible physiological importance. Science 1987; 235: $1043-1046$.

3 Stocker R, Glazer AN, Ames BN: Antioxidant activity of albuminbound bilirubin. Proc Natl Acad Sci USA 1987; 84: 5918-5922.

4 Neuzil J, Stocker R: Free and albumin-bound bilirubin are efficient co-antioxidants for alpha-tocopherol, inhibiting plasma and low density lipoprotein lipid peroxidation. J Biol Chem 1994; 269: 16712-16719.

5 Dudnik LB, Khrapova NG: Characterization of bilirubin inhibitory properties in free radical oxidation reactions. Membr Cell Biol 1998; 12: 233-240.

6 Wu TW, Fung KP, Wu J, Yang CC, Weisel RD: Antioxidation of human low density lipoprotein by unconjugated and conjugated bilirubins. Biochem Pharmacol 1996; 51: 859-862.

7 Dore S, Takahashi M, Ferris CD, Hester LD, Guastella D, Snyder $\mathrm{SH}$ : Bilirubin, formed by activation of heme oxygenase-2, protects neurons against oxidative stress injury. Proc Natl Acad Sci USA 1999; 96: $2445-2450$.

8 Wu TW, Wu J, Li RK, Mickle D, Carey D: Albumin-bound bilirubins protect human ventricular myocytes against oxyradical damage. Biochem Cell Biol 1991; 69: 683-688.

9 Siow RC, Sato H, Mann GE: Heme oxygenase-carbon monoxide signalling pathway in atherosclerosis: anti-atherogenic actions of bilirubin and carbon monoxide? Cardiovasc Res 1999; 41: $385-394$.

10 Mietus-Snyder M, Friera A, Glass CK, Pitas RE: Regulation of scavenger receptor expression in smooth muscle cells by protein kinase C: a role for oxidative stress. Arterioscler Thromb Vasc Biol 1997; 17: 969-978.

11 Amit Y, Boneh A: Bilirubin inhibits protein kinase $\mathrm{C}$ activity and protein kinase C-mediated phosphorylation of endogenous substrates in human skin fibroblasts. Clin Chim Acta 1993; 223: $103-111$.

12 Schwertner HA, Jackson WG, Tolan G: Association of low serum concentration of bilirubin with increased risk of coronary artery disease. Clin Chem 1994; 40: 18-23.

13 Hunt SC, Wu LL, Hopkins PN, Williams RR: Evidence for a major gene elevating serum bilirubin concentration in Utah pedigrees. Arterioscler Thromb Vasc Biol 1996; 16: 912-917.

14 Hunt SC, Kronenberg F, Eckfeldt JH, Hopkins PN, Myers RH, Heiss $\mathrm{G}$ : Association of plasma bilirubin with coronary heart disease and segregation of bilirubin as a major gene trait: The NHLBI Family Heart Study. Atherosclerosis 2001; 154: 747 - 754.

15 Breimer LH, Wannamethee G, Ebrahim S, Shaper AG: Serum bilirubin and risk of ischemic heart disease in middle-aged British men. Clin Chem 1995; 41: 1504-1508.

16 Hopkins PN, Wu LL, Hunt SC, James BC, Vincent GM, Williams RR: Higher serum bilirubin is associated with decreased risk for early familial coronary artery disease. Arterioscler Thromb Vasc Biol 1996; 16: 250-255.

17 Levinson SS: Relationship between bilirubin, apolipoprotein B, and coronary artery disease. Ann Clin Lab Sci 1997; 27: 185-192.

18 Djousse L, Levy D, Cupples LA, Evans JC, D'Agostino RB, Ellison RC: Total serum bilirubin and risk of cardiovascular disease in the Framingham offspring study. Am J Cardiol 2001; 87: 1196-1200.

19 Madhavan M, Wattigney WA, Srinivasan SR, Berenson GS: Serum bilirubin distribution and its relation to cardiovascular risk in children and young adults. Atherosclerosis 1997; 131: 107-113.

20 Kort EN, Ballinger DG, Ding W et al: Evidence of linkage of familial hypoalphalipoproteinemia to a novel locus on chromosome 11q23. Am J Hum Genet 2000; 66: 1845-1856.

21 Williams RR, Hunt SC, Barlow GK et al: Health family trees: a tool for finding and helping young family members of coronary and cancer prone pedigrees in Texas and Utah. Am J Public Health 1988; 78: $1283-1286$. 
22 Routh JI: Liver Function; in Tietz NW (ed) Fundamentals of Clinical Chemistry. Philadelphia: WB Saunders, 1976, pp 1037-1040.

23 O'Connell JR, Weeks DE: PedCheck: A program for identification of genotype incompatibilities in linkage analysis. Am J Hum Genet 1998; 63: 259-266.

24 Lander ES, Green P: Construction of multilocus genetic linkage maps in humans. Proc Natl Acad Sci USA 1987; 84: 2363-2367.

25 Hasstedt SJ.PAP: pedigree analysis package, rev. 4. Salt Lake City, Utah: Department of Human Genetics, University of Utah, 1993.

26 Göring HH, Terwilliger JD: Linkage analysis in the presence of errors I: complex-valued recombination fractions and complex phenotypes. Am J Hum Genet 2000; 66: 1095-1106.

27 Thomas A, Gutin A, Abkevich V, Bansal A: Multilocus linkage analysis by blocked Gibbs sampling. Statistics Comput 2000; 10: $259-269$.

28 Camp NJ, Gutin A, Abkevich V, Farnham JM, Cannon-Albright L, Thomas A: A new nonparametric linkage statistic for mapping both qualitative and quantitative trait loci. Genet Epidemiol 2001; 21 (Suppl 1): S461 -S466.

29 Kruglyak L, Daly MJ, Reeve-Daly MP, Lander ES: Parametric and nonparametric linkage analysis: a unified multipoint approach. Am J Hum Genet 1996; 58: 1347 - 1363.

30 Stone S, Abkevich V, Hunt SC et al: A major predisposition locus for severe obesity, at 4p15-p14. Am J Hum Genet 2002; 70: 14591468.

31 Schwertner HA: Association of smoking and low serum bilirubin antioxidant concentrations. Atherosclerosis 1998; 136: 383-387.

32 Cerne D, Ledinski G, Kager G, Greilberger J, Wang X, Jurgens G: Comparison of laboratory parameters as risk factors for the presence and the extent of coronary or carotid atherosclerosis: the significance of apolipoprotein B to apolipoprotein all ratio. Clin Chem Lab Med 2000; 38: 529-538.

33 Temme EH, Zhang J, Schouten EG, Kesteloot H: Serum bilirubin and 10-year mortality risk in a Belgian population. Cancer Causes Control 2001; 12: 887-894.

34 Vigne P, Feolde E, Ladoux A, Duval D, Frelin C: Contributions of NO synthase and heme oxygenase to cGMP formation by cytokine and hemin treated brain capillary endothelial cells. Biochem Biophys Res Commun 1995; 214: 1-5.

35 Siow RC, Ishii T, Sato $\mathrm{H}$ et al: Induction of the antioxidant stress proteins heme oxygenase- 1 and MSP23 by stress agents and oxidised LDL in cultured vascular smooth muscle cells. FEBS Lett 1995; 368: 239-242.

36 Paganga G, Rice-Evans C, Rule R, Leake D: The interaction between ruptured erythrocytes and low-density lipoproteins. FEBS Lett 1992; 303: 154-158.

37 Nakagami T, Toyomura K, Kinoshita T, Morisawa S: A beneficial role of bile pigments as an endogenous tissue protector: anticomplement effects of biliverdin and conjugated bilirubin. Biochim Biophys Acta 1993; 1158: 189-193.

38 Willis D, Moore AR, Frederick R, Willoughby DA: Heme oxygenase: a novel target for the modulation of the inflammatory response. Nat Med 1996; 2: 87-90.

39 Harding D, Jeremiah SJ, Povey S, Burchell B: Chromosomal mapping of a human phenol UDP-glucuronosyltransferase, GNT1. Ann Hum Genet 1990; 54: 17-21.
40 Bosma PJ, Chowdhury NR, Goldhoorn BG et al: Sequence of exons and the flanking regions of human bilirubin-UDP-glucuronosyltransferase gene complex and identification of a genetic mutation in a patient with Crigler-Najjar syndrome, type I. Нераtology 1992; 15: $941-947$.

41 Ritter JK, Yeatman MT, Ferreira P, Owens IS: Identification of a genetic alteration in the code for bilirubin UDP-glucuronosyltransferase in the UGT1 gene complex of a Crigler-Najjar type I patient. J Clin Invest 1992; 90: 150-155.

42 Moghrabi N, Clarke DJ, Burchell B, Boxer M: Cosegregation of intragenic markers with a novel mutation that causes CriglerNajjar syndrome type I: implication in carrier detection and prenatal diagnosis. Am J Hum Genet 1993; 53: 722 - 729.

43 Bosma PJ, Goldhoorn B, Oude Elferink RP, Sinaasappel M, Oostra BA, Jansen PL: A mutation in bilirubin uridine $5^{\prime}$-diphosphateglucuronosyltransferase isoform 1 causing Crigler-Najjar syndrome type II. Gastroenterology 1993; 105: 216-220.

44 Moghrabi N, Clarke DJ, Boxer M, Burchell B: Identification of an A-to-G missense mutation in exon 2 of the UGT1 gene complex that causes Crigler-Najjar syndrome type 2. Genomics 1993; 18: $171-173$.

45 Owens D, Evans J: Population studies on Gilbert's syndrome. $J$ Med Genet 1975; 12: 152-156.

46 Bailey A, Robinson D, Dawson AM: Does Gilbert's disease exist? Lancet 1977; 1: 931-933.

47 Sieg A, Arab L, Schlierf G, Stiehl A, Kommerell B: Die Prävalenz des Gilbert-Syndroms in Deutschland. Dtsch Med Wochenschr 1987; 112: 1206-1208.

48 Clarke DJ, Moghrabi N, Monaghan G et al: Genetic defects of the UDP-glucuronosyltransferase-1 (UGT1) gene that cause familial non-haemolytic unconjugated hyperbilirubinaemias. Clin Chim Acta 1997; 266: 63-74.

49 Bosma PJ, Chowdhury JR, Bakker C et al: The genetic basis of the reduced expression of bilirubin UDP- glucuronosyltransferase 1 in Gilbert's syndrome. N Engl J Med 1995; 333: 1171-1175.

50 Monaghan G, Ryan M, Seddon R, Hume R, Burchell B: Genetic variation in bilirubin UPD-glucuronosyltransferase gene promoter and Gilbert's syndrome. Lancet 1996; 347: 578-581.

51 Iolascon A, Faienza MF, Centra M, Storelli S, Zelante L, Savoia A: (TA) 8 allele in the UGT1A1 gene promoter of a Caucasian with Gilbert's syndrome. Haematologica 1999; 84: 106-109.

52 Vitek L, Jirsa $\mathrm{M}$, Brodanova $\mathrm{M}$ et al: Gilbert syndrome and ischemic heart disease: a protective effect of elevated bilirubin levels. Atherosclerosis 2002; 160: 449-456.

53 Cali JJ, Russell DW: Characterization of human sterol 27-hydroxylase. A mitochondrial cytochrome P-450 that catalyzes multiple oxidation reaction in bile acid biosynthesis. J Biol Chem 1991; 266: 7774-7778.

54 Samuels M, Forbey K, Reid J et al: Identification of a common variant in the lipoprotein lipase gene in a large Utah kindred ascertained for coronary heart disease: the $-93 \mathrm{G} / \mathrm{D} 9 \mathrm{~N}$ variant predisposes to low HDL-C/high triglycerides. Clin Genet 2001; 59: $88-98$.

55 Hixson JE, Almasy L, Cole S et al: Normal variation in leptin levels in associated with polymorphisms in the proopiomelanocortin gene, POMC. J Clin Endocrinol Metab 1999; 84: 3187-3191. 